\title{
ARTÍCULOS
}

\section{DILEMAS Y TENSIONES DE LA PRÁCTICA DOCENTE CON ALUMNADO MIGRANTE*}

\section{DILEMMAS AND TENSIONS TEACHERS FACE WITH MIGRANT STUDENTS}

\author{
Pilar Cucalón Tirado ${ }^{1}$ \\ CONICET/Universidad Nacional de La Plata \\ Margarita del Olmo² \\ Instituto de Lengua, Literatura y Antropología, CSIC
}

Recibido: 22 de enero de 2018; Aprobado: 22 de mayo de 2018; Publicado online: 28 mayo de 2019

Cómo citar este artículo / Citation: Cucalón Tirado, Pilar y Margarita del Olmo. 2019. «Dilemas y tensiones de la práctica docente con alumnado migrante». Disparidades 74(1): e007. doi: <https://doi.org/10.3989/dra.2019.01.007>.

RESUMEN: En este texto abordaremos los dilemas y tensiones a los que se enfrenta el profesorado de un programa dirigido al alumnado migrante recién llegado a la Comunidad de Madrid (España). El análisis surge de la oportunidad de enlazar nuestros trabajos de campo etnográficos, que han sido llevados a cabo en dos momentos diferentes de la historia del mencionado programa. El diálogo entre ambas investigaciones nos ha permitido una comprensión más compleja de las prácticas docentes. Especialmente, aquellas que orientan al alumnado migrante hacia itinerarios formativos que no son los regulares para acceder a la universidad, siendo contradictorio con las altas expectativas que tienen dichos estudiantes. Podemos concluir que los argumentos y estrategias utilizadas por el profesorado deben entenderse en el marco de una escuela con serias limitaciones para producir la igualdad de oportunidades que dice preferir.

PALABRAS CLAVE: Antropología de la educación; Etnografía escolar; Políticas de atención a la diversidad; Alumnado migrante; Prácticas docentes; Comunidad de Madrid; Aulas de Enlace.

\begin{abstract}
In this text, the authors address the dilemmas and tensions teachers face in the context of a program designed for migrant students who have recently arrived in the Community of Madrid region of Spain. The analysis combines both authors' ethnographic fieldwork, carried out independently and at different times in the program's history. The dialogue between both research studies provides a more nuanced understanding of teaching practice, especially when schools steer students towards particular educational itineraries - away from university and into vocational training - in conflict with students' higher expectations for their own academic performance. Authors conclude by stating that teachers' strategies and arguments should be understood within the framework of a school with significant limitations to provide all students with the equal opportunities that the school proclaims.
\end{abstract}

KEYWORDS: Anthropology of Education; School Ethnography; Diversity Policies; Migrant Students; Teaching Practice; Community of Madrid; Linking Classrooms.

Copyright: (C) 2019 CSIC. Este es un artículo de acceso abierto distribuido bajo los términos de la licencia de uso y distribución Creative Commons Reconocimiento 4.0 Internacional (CC BY 4.0).

* Una primera versión mucho más reducida de este texto la presentamos con el título "Las Aulas de Enlace: un dispositivo para gestionar la diversidad cultural en la Comunidad de Madrid», en el simposium Migraciones y Educación: Miradas Etnográficas del I Congreso Internacional de Antropología AIBR que se celebro en la Universidad Autónoma de Madrid 7-10 de julio de 2015. Queremos agradecer especialmente las lecturas críticas del borrador que han hecho Silvina Cordero, Ana Dumrauf, Carmen Osuna y Jorge Rolland, sus comentarios y sugerencias han enriquecido significativamente la versión final del texto.

1 Correo electrónico: pilarcucalon82@gmail.com. ORCID iD: <https://orcid.org/0000-0002-5244-7280>.

2 Correo electrónico: margarita.delolmo@cchs.csic.es. ORCID iD: <https://orcid.org/0000-0002-2968-5432>. 
"he visto llorar a alumnos que sacaban menos nota de lo que estaban acostumbrados en su país de origen" (Profesora 2 del Aula de Enlace en un Instituto de Enseñanza Secundaria en Parla, entrevista realizada por Pilar Cucalón Tirado, 11 jun. 2009)

\section{INTRODUCCIÓN}

Este texto tiene la intención de abordar los dilemas y las tensiones que vive el profesorado en su práctica docente con alumnado migrante en el contexto de un programa educativo diseñado específicamente para facilitar la transición de este alumnado al sistema educativo de la Comunidad de Madrid. La posibilidad de centrar nuestra atención en el análisis de los dilemas y las tensiones ha sido resultado de la combinación de nuestros respectivos trabajos de campos etnográficos, realizados tanto de forma independiente como en momentos distintos. Esta combinación nos ha permitido, por un lado, ampliar las perspectivas de análisis en términos de tiempo y espacio pero, sobre todo, construir una conversación que ha enriquecido nuestras investigaciones individuales, ofreciendo más detalle y más complejidad en relación con el objetivo de este artículo.

Nos hemos planteado abordar dicho objetivo para comprender la práctica del profesorado de una forma más integral, dado que en nuestras publicaciones anteriores no habíamos profundizado tanto en este tema. Pero también se nos presentaba como estrictamente necesario para entender los procesos que podían estar influyendo en el hecho de que un alto porcentaje de estudiantes de las Aulas de Enlace, dispositivo en el que llevamos a cabo nuestras investigaciones, esté siendo dirigido por el profesorado del programa y profesionales de los equipos de orientación psicopedagógica y de dirección hacia itinerarios formativos que funcionan en los límites del sistema educativo, y desde los que difícilmente se puede acceder a la universidad (Ávila y Malo 2007; Pérez Milans 2007; Poveda, Jociles y Franzé 2009; Cucalón y Del Olmo 2010; Carrasco y Gibson 2010; Carrasco, Pàmies y Narciso 2011). Visibilizar esta situación siempre ha sido especialmente importante para nosotras porque estamos dando cuenta de las desiguales oportunidades que las escuelas ofrecen al alumnado extranjero. Sin embargo respecto a trabajos anteriores, nuestra contribución consiste en analizar con mayor profundidad las decisiones y las justificaciones que el personal docente toma con respecto a las trayectorias de los alumnos y alumnas migrantes contextualizadas en un programa que ha sido diseñado para facilitar su transición.

Tras presentar brevemente algunas de las características de nuestros trabajos de campo, vamos a comenzar introduciendo el programa, desde su inicio hasta la actualidad, señalando los cambios que nuestras respectivas investigaciones, combinadas, nos permiten apreciar como significativos. A continuación exploraremos la idea del programa como espacio de acogida desde la perspectiva de las orientaciones metodológicas, así como a partir de la percepción de profesores y estudiantes. Todo ello nos permitirá abordar las tensiones y dilemas a los que se enfrenta el profesorado que trabaja con el alumnado migrante de las Aulas de Enlace con la finalidad de ofrecer explicaciones respecto a un tema que nos preocupa especialmente: la contradicción entre la alta valoración de algunos de sus estudiantes y las bajas expectativas con las que orientan sus itinerarios formativos posteriores.

\section{SOBRE NUESTROS TRABAJOS DE CAMPO}

Las dos autoras hemos realizado nuestros trabajos de campo etnográficos en Aulas de Enlace. Las Aulas de Enlace forman parte de un programa propuesto por la Comunidad de Madrid que tiene como objetivo facilitar la incorporación del alumnado migrante recién llegado al sistema educativo de la región. El programa es similar, aunque no idéntico, a otros puestos en marcha en territorios del estado español, tales como Aules d'Acollida en Cataluña (Carbonell 2006; Alegre, Benito y González 2008; Vila et al. 2009), Aulas Temporales de Adaptación Lingüística (ATAL) en Andalucía (Jiménez, Cotrina y García 2011; Ortiz Cobo 2011; Antolínez 2015; Castilla 2015; García, Castilla y Capellán 2015; Etxeberria et al. 2015) o Aulas de acogida en Murcia (Escarbajal Frutos 2010). En todos los casos se trata de programas de adaptación para estudiantes nacidos fuera del Estado español, tienen duraciones temporales, pueden o no estar alternados con las clases regulares, y se centran en la adaptación lingüística y académica del alumnado.

En la Comunidad de Madrid, el programa se incluyó en un «Glosario de medidas de atención a la diversidad» en el que se encontraban listadas 29 actuaciones o programas, algunas de las cuales tenían un origen anterior al concepto político-educativo de «atención a la diversidad en Educación». La mayor parte de estas 
medidas estaban orientadas desde la filosofía de la "compensación», es decir dirigidas a una población escolar que se desvía de la norma y cuyo objetivo es "compensar» las carencias del alumnado que se aparta del modelo, tales como «necesidades educativas especiales asociadas a condiciones sociales y culturales desfavorecidas», «desfase curricular», «riesgo de abandono escolar» o "desconocimiento de la lengua castellana» ${ }^{3}$. Algunos y algunas autoras han señalado el hecho de que estas medidas dedicadas a «atender la diversidad» en realidad proceden de medidas anteriores dedicadas específicamente al alumnado gitano (Mijares Molina 2002; García, Rubio y Bouachra 2011) o están relacionadas «con la escolarización de hijos e hijas de grupos minoritarios y minorizados, es decir, aquellos que ocupan posiciones permanentemente subalternas en términos sociales y culturales» (Carrasco 2011: 273). Sin embargo, a diferencia del resto de las «medidas de atención a la diversidad», las Aulas de Enlace fueron diseñadas específicamente para atender las necesidades del alumnado migrante. Por este motivo creemos que son un espacio privilegiado para analizar las tensiones y los dilemas de la práctica de los y las docentes y el empleo explícito de las ideas que atribuyen a sus culturas de procedencia en los discursos con los que legitiman sus decisiones y sus contradicciones.

Margarita del Olmo desarrolló su investigación durante la puesta en marcha y los primeros años de desarrollo del programa en un colegio concertado (privado pero sostenido con fondos públicos), entre 2005 y 2009, en la ciudad de Madrid (Del Olmo 2010), donde en el curso 2007-2008 el porcentaje de alumnado extranjero era de 16,1 \% según la Consejería de Educación (2009). Concretamente trabajó en el distrito de Latina, en ese momento uno de los distritos de mayor concentración de alumnado migrante. A los largo de los tres cursos escolares realizó observación participante en el aula una vez por semana y entrevistas en profundidad formales a los agentes educativos del colegio y a las personas responsables del diseño y la puesta en marcha del programa en la Consejería de Educación de la Comunidad de Madrid, e informales a los 25 chicos y 14 chicas de 12 a 18 años escolarizados en el aula en los tres cursos que procedían de Brasil (14), Rumanía (13), China (4),

3 Documento de circulación interna de la Consejería de Educación sin fecha consultado por Margarita del Olmo durante su trabajo de campo.
Ucrania (4), Polonia (2), Marruecos (2), Bulgaria (2) y República Dominicana (2). Del ese total de 43, solo cuatro permanecieron en la misma escuela una vez que acabaron el programa en el Aula de Enlace (menos del $10 \%$ ), aunque un quinto del total cursó su primer año en la misma escuela, pero cambió de colegio durante el segundo.

El profesorado a cargo del aula durante el trabajo de campo estuvo compuesto por una tutora titular durante el primer y el tercer año de trabajo de campo, y durante el segundo fue relevada, mientras cumplía una baja médica, por una profesora que había comenzado en el aula como profesora en prácticas. Además de este núcleo central, en la clase entraban otros profesores del colegio a impartir materias específicas o realizar actividades y varias profesoras en prácticas procedentes de programas de formación inicial de profesorado de distintas universidades. La relación con las tutoras, que dieron su permiso explícito a la dirección para la investigación, siempre fue muy cordial y progresivamente más próxima, y durante la última parte del trabajo de campo fueron invitadas a participar como socias en una red europea sobre educación intercultural, financiada por el programa Comenius de la Unión europea. Esta Red proporcionó los fondos necesarios a la autora y a las tutoras para establecer los marcos en los que discutir, compartir y contradecir las ideas de las tres con respecto al tratamiento de la diversidad en la escuela en general y sobre el programa de las Aulas de Enlace en particular. Esa relación fuera del aula también determinó la relación dentro de ella.

Pilar Cucalón Tirado llevó a cabo la suya en el periodo de mayor apogeo de la medida y hasta su drástica disminución, específicamente entre 2008-2009 y 20102011 en un instituto público de $\mathrm{Parla}^{4}$, una pequeña ciudad de la Comunidad de Madrid, y en un colegio concertado también del distrito de Latina en Madrid capital (Cucalón Tirado 2015). En $2011^{5}$ el porcentaje

4 En Parla, según el Instituto de Estadística de la Comunidad de Madrid, estaban censadas 121995 personas en 2011. Ver en <www.madrid.org/desvan/almudena/FichaMunicipal.icm?codMunZona=1065>. Fecha de acceso: 14 abr. 2012.

5 A día de hoy, las cifras de alumnado extranjero se han visto reducidas, aunque no en un porcentaje muy elevado. Durante el curso 2016-2017, en la Comunidad de Madrid había 1179185 estudiantes matriculados en Enseñanzas de Régimen General, de los cuales 127112 eran extranjeros (en porcentajes: el 10,8 \% del total). En el mismo periodo, el 20,2 \% del alumnado de Parla es de otro país, 
de alumnado migrante en la Comunidad de Madrid era de un 13,4 \% y en Parla de un 27,4 \% (Dirección General de Mejora de la Calidad de la Enseñanza 2011), siendo además una de las localidades con la Renta Bruta Per Cápita más baja de la región (11 928,78 euros frente a la media regional que era de 17623,78 euros) ${ }^{6}$. A lo largo del primer curso, realizó observación participante una vez por semana en cada una de las Aulas de Enlace, además de entrevistas en profundidad al profesorado, profesionales de los equipos directivos y de orientación. En el segundo, realizó observaciones en un aula de referencia del colegio concertado en la que habían sido escolarizados antiguos estudiantes de Aula de Enlace. Durante el tercero, entrevistó a ex- alumnos y alumnas del Aula de Enlace del instituto público de Parla y a profesoras de otras Aulas de Enlace de la región.

Durante el primer curso escolar estableció un contacto continuado con 27 chicos y chicas con edades comprendidas entre los 12 y 18 años ${ }^{7}$. Dos cursos más tarde, gracias al trabajo en el instituto público, reconstruyó las trayectorias académicas de 16 (9 chicas y 7 chicos) cuyos países de origen eran Moldavia (1), Marruecos (1), Brasil (1), Polonia (2), Senegal (2) y China (9). Solo dos realizaron estudios de bachillerato, según la información que pudo recoger, aunque le faltan datos respecto a los itinerarios escolares actuales.

En el instituto público había dos profesoras tutoras del Aula de Enlace, una de ellas fue sustituida por enfermedad durante unas semanas en 2008-2009. Durante ese curso, en el colegio concertado fue responsable del Aula de Enlace un docente sustituto de la titular, con esta última tuvo más contacto en el siguiente año. En este centro, además, se incorporaban a la clase diferentes profesionales para impartir materias específicas. La investigación en la escuela pública fue posible porque, tras una presentación previa del proyecto, las docentes lo solicitaron a la dirección. En el caso de la concertada, fue la dirección la que sugirió al profesorado la realización del trabajo de campo. La relación establecida con cada docente fue muy diferente, siendo con una de las profesoras de Parla con la que pudo compartir más análisis e ideas, a pesar de la divergencia de posturas. Con las dos profesoras de

siendo Marruecos la nacionalidad alóctona mayoritaria (Consejería de Educación e Investigación 2017).

6 Ver en <www.madrid.org/desvan/almudena/FichaMunicipal.icm?codMunZona=1065>. Fecha de acceso: 20 abr. 2012.

7 En 2009-2010 no realizó un trabajo tan exhaustivo en el Aula de Enlace del colegio concertado pero pudo conocer a ocho estudiantes más. esta escuela llevó a cabo una actividad de autorretratos en una página web de su autoría, con la intención de que el antiguo alumnado del Aula de Enlace pudiera contar sus experiencias dentro y al salir del programa. En este punto es preciso señalar que se trató de una investigación sobre y en las Aulas de Enlace pero no se planteó ni se desarrolló como un trabajo en colaboración con distintos agentes escolares.

Las tres Aulas de Enlace analizadas por las autoras eran de secundaria y han desaparecido a día de hoy. Sin embargo, como argumentaremos a continuación, el programa continúa funcionando bajo mínimos en otros centros escolares.

Queremos señalar, por lo tanto, que los dos trabajos de campo se realizaron de manera independiente, que cada una de las autoras llevó a cabo su análisis y publicó sus resultados por separado. Sin embargo, nos propusimos revisitar ambos con la intención de entrelazarlos, guiadas por la expectativa de que esta combinación nos permitiría, como hemos señalado, no solo una mayor profundidad temporal, variedad de análisis y sujetos de estudio, sino sobre todo una mayor complejidad a la hora de comprender el tema propuesto.

\section{BREVE REPASO POR LA NORMATIVA QUE REGULA LAS AULAS DE ENLACE}

En el curso 2002-2003 la Comunidad de Madrid puso en marcha un programa dirigido a los estudiantes extranjeros recién llegados a la región para facilitar su incorporación al sistema educativo. El programa se denominó, en un principio, Escuelas de Bienvenida y pretendía regular tres subprogramas: las Aulas de Enlace, el programa de ocio y tiempo libre y la formación del profesorado en educación intercultural. Los dos últimos tuvieron un incipiente desarrollo y muchas dificultades de puesta en marcha, hasta que se terminaron desechando a partir de la reducción de presupuesto. Las Aulas de Enlace son las únicas que permanecen vigentes, pero hoy en día en un número muy reducido.

La medida está dirigida a los estudiantes extranjeros recién llegados que desconocen el castellano y/o presentan desfase curricular. Sus principales objetivos, tal y como aparecen en las normativas que los regulan, son: a) enseñar la lengua vehicular (el castellano), b) acortar el periodo de incorporación al sistema educativo, c) favorecer el desarrollo de su identidad personal y cultural, y d) posibilitar la inte- 
gración en el medio social. El número máximo de estudiantes que pueden reunir es 12 y el mínimo 5 (Consejería de Educación 2008).

El programa puede ser solicitado por los colegios sostenidos con fondos públicos (públicos o privados $\operatorname{concertados}^{8}$ ), pero siempre deben ser recomendados por las Direcciones de Área Territorial. Las escuelas tienen que cumplir una serie de requisitos: 1 ) aceptar los términos del programa; 2) disponer de espacios adecuados; 3 ) contar con plazas para poder matricular al alumnado una vez que salga del Aula de Enlace; 4) pertenecer a zonas geográficas con una concentración de población migrante significativa; 5) tener experiencia con estudiantes extranjeros que desconozcan la lengua vehicular (Consejería de Educación 2008); 6) disponer de comedor y clases extraescolares, y finalmente (y esta condición solo se cumplió al principio); y 7) equilibrar las Aulas de Enlace entre áreas urbanas y rurales ${ }^{9}$.

Existen Aulas de Enlace en primaria (alumnos y alumnas entre 9 y 12 años), en secundaria (entre 12 y 16 años $^{10}$ ) y mixtas (que reúnen a todos y todas indepen-

8 En el sistema educativo español existen tres tipos de escuelas: privadas, públicas y concertadas. Las escuelas privadas pertenecen a personas o instituciones privadas, se regulan de manera autónoma y se financian, en términos generales, a partir de las matrículas que pagan las familias por el alumnado. Las escuelas públicas dependen económicamente y están reguladas por el estado de cada comunidad autónoma: el profesorado se contrata a partir de un examen público organizado y su sueldo también procede de los fondos del Estado. Las escuelas concertadas es un sistema mixto que combina características de la gestión privada y de la pública, firman un contrato con el estado que les compromete a seguir la misma regulación que las escuelas públicas y por el que reciben financiación estatal, pero conservan una gestión privada que les permite contratar al profesorado y gestionar sus sueldos a partir del dinero procedente del Estado; el alumnado no debe pagar matrícula aunque, en la mayoría de las ocasiones, se exige una pequeña cuota a las familias por diversos conceptos y razones.

9 Estas dos últimas condiciones están en las normativas anteriores a 2008.

10 La enseñanza es obligatoria en España hasta los 16 años, que corresponden con 4. curso de la ESO (Educación Secundaria Obligatoria). Tras la obligatoriedad, el alumnado puede cursar dos años de Bachillerato, que es el itinerario que dirige a la universidad, o los niveles medio y superior de Formación profesional. Aunque a esta norma, la última ley de educación, LOMCE (Ley Orgánica para la Mejora de la Calidad Educativa), aprobada en 2013, introdujo la novedad de que el itinerario de Formación Profesional se puede iniciar al finalizar 3.o de la ESO. Las Aulas de Enlace se dirigen a los alumnos y alumnas hasta los 16 años o mientras cursan la educación obliga- dientemente de su edad). Al principio las mixtas eran muy poco numerosas y constituían la excepción más que la regla; sin embargo, hoy día sucede al contrario en la ciudad de Madrid ${ }^{11}$, a pesar de que las aulas mixtas fueron desaconsejadas por las personas de la propia Consejería que evaluaron su funcionamiento. Como argumentaremos en el siguiente apartado, desde nuestro punto de vista, el aumento de las Aulas de Enlace mixtas responde tanto a una reducción de presupuesto como a la progresiva privatización del programa.

En cuanto al tiempo de permanencia, el alumnado puede asistir un máximo de nueve meses, aunque es posible prorrogarlo. Este periodo de nueve meses es el tiempo que la Consejería de Educación considera necesario para que se incorporen a las clases ordinarias con un nivel de castellano adecuado. El profesorado, junto a los equipos de orientación y siguiendo las instrucciones de la Administración, es quien aconseja el curso o programa (Compensatoria, Diversificación, etcétera) en el que los y las estudiantes serán escolarizados una vez finalicen. Utilizan como criterios tanto el desenvolvimiento en castellano como el desfase curricular (Consejería de Educación 2008).

Durante su estancia en el programa, el alumnado debe incorporarse simultáneamente a las aulas que le corresponden por edad en algunas asignaturas. La normativa aconseja que sea en Educación física, Educación plástica y visual, Música y Tecnologías, ya que las considera más propicias para el establecimiento de las relaciones con sus compañeros y compañeras; sin embargo, el profesorado tiene la posibilidad de recomendar que se amplíe el número de asignaturas en función de la motivación de los chicos y chicas (Consejería de Educación 2008).

\section{CAMBIOS EN EL FUNCIONAMIENTO DE LAS AULAS DE ENLACE: HACIA UN PROCESO DE PRIVATIZACIÓN}

En el apartado anterior hemos explicado de forma general las instrucciones que regulan el funciona-

toria; sin embargo, en el programa hemos encontrado estudiantes que ya habían cumplido los 18 años.

11 A 3 de abril de 2017 había 29 Aulas de Enlace mixtas, 6 en primaria y 5 en secundaria (<http://www.madrid.org/dat_capital/bienvenida/impresos_pdf/AEnlaceCapital1617.pdf>, Fecha de acceso: 22 ago. 2017). No podemos ofrecer información sobre el resto de la Comunidad dado que no está disponible ni hemos conseguido que la Consejería nos la facilitara. 
miento de las Aulas de Enlace. El presente análisis nos ha posibilitado ampliar el periodo de estudio del programa y por lo tanto reconocer los cambios que ha ido sufriendo la medida, entendiéndolos como un proceso que nos permite analizarlo con mayor profundidad y riqueza, descubriendo las intencionalidades que vistas de manera puntual resultan más difíciles de identificar y de entender. Para ilustrar aquí ese proceso vamos a emplear como argumentos las fuertes variaciones en la normativa que regula el programa, la evolución de la cantidad de clases y su distribución con respecto a la titularidad de centro ${ }^{12}$.

El primer cambio que queremos señalar es el hecho de que al principio las Aulas de Enlace formaban parte de un programa más comprensivo denominado Escuelas de Bienvenida que incluían actividades de ocio y tiempo y libre y formación intercultural para los y las docentes.

El subprograma de ocio y tiempo libre consistía en realizar actividades lúdicas con todo el alumnado del colegio en las que se promovía que participaran las chicas y chicos del Aula de Enlace. Al principio estas actividades se programaron para el fin de semana pero enseguida se llevaron a cabo en horario escolar por la oposición de los y las docentes a ampliar su horario de trabajo sin una remuneración extra. Asimismo, se convirtieron en actividades elegidas por el profesorado, financiadas por el programa, pero encargadas a empresas o asociaciones, siendo este uno de los primeros pasos en el proceso de externalización de la atención a la diversidad y progresiva privatización del programa (Laval 2005; Aguado, Mata y Gil-Jaurena 2017).

El programa de formación intercultural para docentes responsables de las Aulas de Enlace nunca se realizó, se sustituyó desde el principio por una reunión anual en la que el personal responsable de la Consejería explicaba los cambios anuales que iban introduciendo en la medida. La formación permanente del profesorado quedó limitada al programa general que también se ha ido reduciendo progresivamente.

Durante 2008 se establece, por primera vez, un número mínimo de estudiantes para que el programa

12 Este análisis está basado en las cifras que ofrece publicada la Consejería de Educación cada año (Consejería de Educación 2009, 2010a y 2010b; Consejería de Educación y Empleo 2012; Consejería de Educación, Juventud y Deporte 2013, 2014 y 2015). pueda continuar en una escuela; anteriormente, sin embargo, solo se señalaba el máximo de plazas por aula. Otro aspecto que consideramos relevante es que utiliza por primera vez el término «carencia» para referirse al desconocimiento del castellano (aunque dicho término ya había aparecido en normativas anteriores en relación a los conocimientos básicos). Además, queremos señalar que ese mismo año desaparece la Dirección General de Promoción Educativa en la Consejería de Educación ${ }^{13}$ que se encargaba del impulso y seguimiento del programa. A partir de ese momento la gestión del mismo queda subdividida y desmembrada entre la Dirección General de Educación Infantil y Primaria, la Dirección General de Educación Secundaria, la Dirección General de Becas y Ayudas y las cinco direcciones de Área Territorial, haciendo mucho más difícil la coordinación y por lo tanto su coherencia ${ }^{14}$.

En relación con la cantidad de las Aulas de Enlace puede apreciarse una reducción significativa desde su comienzo hasta el curso actual, de tal modo que en el 2002-2003 había 132 y en el 2016-2017 solo quedan 75. Aunque esta cifra ofrece aún mayor contraste al compararla con las 293 del curso 2008-2009, que es cuando alcanzaron un número más elevado, y supone el momento en el que empiezan a descender. De todos modos, hemos observado que en el curso 2016-2017 los informes dan cuenta de un aumento significativo.

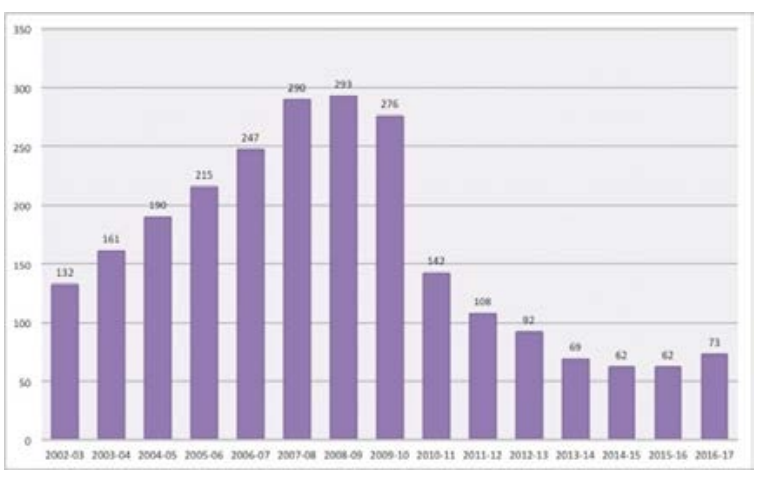

Gráfico 1.- Cantidad de Aulas de Enlace en la Comunidad de Madrid $^{15}$

13 Utilizamos esta denominación de Consejería de Educación de una manera genérica, dadas las diversas fluctuaciones que ha vivido en su denominación y sus competencias.

14 Esta situación nos ha afectado directamente como investigadoras, porque la desmembración del programa ha incrementado las dificultades de relación con cada una de estas instancias, que a su vez se acaba traduciendo en una mayor opacidad de los datos.

15 Elaborado por Pilar Cucalón basada en datos de la Consejería de Educación de la Comunidad de Madrid. Ver en 
Nos parece necesario resaltar la evolución de la distribución de las Aulas de Enlace entre centros públicos y concertados. En el inicio (2002-2003), 67 estaban emplazadas en centros públicos y 65 en concertados. A partir del curso 2011-2012 la tendencia de distribución ha dado la vuelta, de manera que en el curso 2016-2017, 27 aulas están localizadas en escuelas públicas y 46 en concertadas. Esta distribución no se encuentra apoyada por la presencia del alumnado extranjero, sino todo lo contrario, ya que el $14,4 \%$ del total está escolarizado en la enseñanza pública y el 6,6 \% en la concertada ${ }^{16}$ (datos de la Consejería de Educación). Por otro lado, este proceso ha implicado una presencia destacada de las aulas mixtas, al menos en la ciudad de Madrid: en abril de 2017, de un total de 40 clases en la capital, 29 eran mixtas, 6 de primaria y 5 de secundaria. Esta evolución resulta especialmente chocante en relación con las conclusiones de la evaluación que hizo en su día la Subdirección General de Inspección Educativa, recomendando la desaparición de la mixtas por su carácter excesivamente heterogéneo ${ }^{17}$.

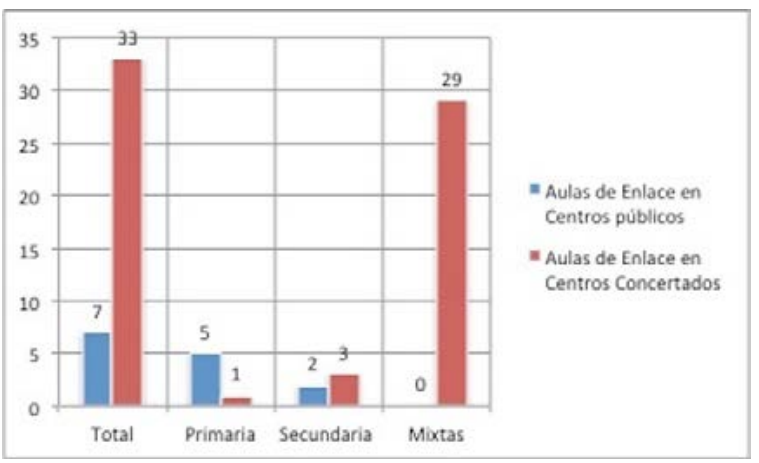

GráfIco 2.- Cantidad de Aulas de Enlace en Madrid capital ${ }^{18}$

«Datos y Cifras de la Educación» 2002-2003, 2003-2004, 2004-2005, 2005-2006, 2006-2007, 2007-2008, 2008-2009, 2009-2010, 2010-2011, 2011-2012, 2012-2013, 2013-2014, 2014-2015, 2015-2016, 2016-2017, en <http://www.madrid.org/cs/Satellite?c=CM_InfPractica_FA\&cid $=11425584$ 05036\&language $=$ es\&pagename $=$ ComunidadMadrid\%2FEs tructura\#subir> Fecha de acceso: 25 ago. 2017.

16 Sobre la distribución del alumnado migrante entre centros públicos y concertados en la ciudad de Madrid, véase el análisis de Poveda, Franzé, Jociles et al. (2011).

17 Las evaluaciones se realizaron para tres cursos escolares consecutivos: 2002-2003, 2003-2004 y 2004-2005. Aunque el informe de evaluación no se ha hecho nunca público, la Subdirección General de Inspección educativa permitió hacer una consulta de sus conclusiones en la sede de la Subdirección a Margarita del Olmo el 11 de mayo de 2007, que además realizó una entrevista a una de las personas responsables en Madrid el 8 de marzo de 2007 (Del Olmo 2009).

18 Elaborado por Pilar Cucalón Tirado basada en datos de la Consejería de Educación de la Comunidad de Madrid. Ver
Este último dato nos parece muy significativo, ya que en las aulas mixtas se reduce la cantidad de profesorado dedicado al programa y con ello la propia inversión, puesto que donde antes había dos clases, una de primaria y otra de secundaria, ahora hay solo una. Además, los centros públicos tenían asignados dos docentes por clase pero los concertados uno o una. Los centros públicos, por su división entre primaria y secundaria, no pueden tener aulas mixtas.

A la luz de los argumentos aportados, concluimos que las variaciones documentadas no responden simplemente al hecho de que se vayan «mejorando cosas sobre la marcha», como dijo en su día María Antonia Casanova (Tobarra 2006), responsable del Programa Escuelas de Bienvenida hasta el año 2007. Al menos no lo vemos tan claramente a partir del curso 20082009 cuando la normativa experimenta las transformaciones que hemos mencionado. Tampoco consideramos que los cambios acontecidos a partir de 2011-2012 se reduzcan a la disminución del número de Aulas de Enlace, sino que comienza a prevalecer el modelo de Aula de Enlace mixta en los centros concertados. Este dato podría pensarse como el modo de equilibrar la escolarización del alumnado migrante entre centros públicos y concertados, una reivindicación de los sindicatos docentes ${ }^{19}$, pero las cifras disponibles no muestran tal redistribución. Dicha situación, desde nuestro punto de vista, muestra un pequeño paso más en el proceso de privatización de la escuela pública (Aguado, Mata, Gil-Jaurena 2017), dado que los centros públicos cuentan con un recurso menos. Como nos indicaban lúcidamente una antigua profesora de Aula de Enlace y una Técnica de Servicios a la Comunidad de un Instituto de Enseñanza Secundaria (IES) de la Sierra Norte de Madrid en el que Pilar Cucalón Tirado realizó algunas entrevistas:

Es interesante saber las cifras de Aulas de Enlace, pero hay que tener en cuenta que aunque el Aula de Enlace esté como tal (exista, quería decir) es posible que no esté funcionando todo el horario o que haya menos profesores (Extracto del diario de

en <https://www.educa2.madrid.org/web/educamadrid/ principal/files/95ef3aac-ead3-44d6-8935-7996c8fc61f5/ AEnlaceCapital.pdf?t=1499415887634> Fecha de acceso: 17 oct. 2018.

19 Una reivindicación que podríamos considerar legítima teniendo en cuenta que ambos tipos de centros son mantenidos con fondos públicos, pero es preciso señalar que está contribuyendo a la producción de un discurso que problematiza a los niños, niñas y jóvenes migrantes, como si su mera presencia fuera negativa en la enseñanza. 
campo de Pilar Cucalón Tirado, Instituto de Enseñanza Secundaria en la Sierra Norte de Madrid, 1 mar. 2011).

En los centros concertados donde hicimos trabajo de campo ya se producía esta situación donde solo un o una docente se encargaba del Aula de Enlace aunque el profesorado del resto del colegio también pudiese impartir clase algunas horas a la semana.

Para finalizar querríamos subrayar que atender a estas variaciones nos resulta fundamental por dos razones. En primer lugar, para contribuir a las investigaciones que vienen desarrollando análisis minuciosos sobre la trayectoria de este tipo de programas educativos (Antolínez 2015; Castilla 2015; Etxeberria et al. 2015; García, Castilla y Capellán 2015) ${ }^{20}$ lo cual responde, a su vez, a los periodos tan diferentes en los que llevamos a cabo nuestros trabajos; y en segundo lugar, para dar cuenta de otra de las tensiones que sufrían los y las docentes del programa con quienes tuvimos contacto, un aspecto relevante que nos permite comprender de forma más compleja sus prácticas e interpretaciones.

\section{LAS AULAS DE ENLACE COMO ESPACIOS DE ACOGIDA}

Las normativas relativas a las Aulas de Enlace nunca hacen alusión al tema de la acogida. Sin embargo, un libro encargado por la Consejería de Educación, realizado con la colaboración de docentes de Aula de Enlace, señala que no se tratan solo de un lugar de aprendizaje de la lengua o de trabajo sobre el desfase curricular, sino de un programa en el que el estudiante debería: «ser reconocido por lo que es y lo que sabe, comprender sus sentimientos de extrañeza, manifestarle nuestra disposición a escuchar y dar lugar a sus experiencias» (Boyano et al. 2006: 35).

Una de las autoras del libro, entrevistada por Pilar Cucalón Tirado el 24 de marzo de 2011, enfatizó que las Aulas de Enlace habían sido concebidas como un espacio de "acogida» para el alumnado migrante recién llegado, dado que el programa de compensatoria, donde solía ser escolarizado en primer lugar, no lo consideraba adecuado teniendo en cuenta las ca-

20 Estos trabajos se presentaron al VIII Congreso de Migraciones Internacionales en España, en el simposio Aulas especiales para la acogida de inmigrantes, celebrado en Granada en 2015. racterísticas atribuidas a niños, niñas y jóvenes. Relacionaba la «acogida» con el desarraigo. Un aspecto que docentes de nuestros trabajos de campo también resaltaban:

pues me está gustando, te voy a decir por qué. Porque son chicos que creo que llevan por detrás de sí una historia familiar muy arraigada a un sitio, tienen ese desarraigo y se tienen que buscar anclas aquí (Profesora de Aula de Enlace de un Colegio Concertado en Madrid, entrevista realizada por Pilar Cucalón Tirado, 2 jun. 2009).

En el siguiente extracto de entrevista podrá verse como dicha "acogida» es considerada por otro docente como un paso necesario a la incorporación posterior a las aulas de referencia, que define como lugares «locos»:

El Aula es una forma de que les cueste menos llegar a un sitio tan loco para ellos, tan distinto y cumple su función pero la integración es complicada (Profesor de aula de referencia de un Instituto de Enseñanza Secundaria en Parla, entrevista realizada por Pilar Cucalón Tirado, 29 jun. 2009).

Por otro lado, la Consejería de Educación, al evaluar la medida recogió las siguientes opiniones entre directores y directoras de centros, profesores y profesoras de Aula de Enlace, resto de docentes del colegio y alumnos y alumnas (Del Olmo 2009): que el alumnado está muy satisfecho, contento y agradecido; que el clima de trabajo es muy agradable; que el alumnado se mostraba participativo y colaborador en el aula y en las actividades de la vida del centro; que tenía un alto concepto del profesorado; que supone un medio de integración que contribuye a darle un marco de seguridad y que por el contrario, cuando tiene que salir del programa, manifiesta gran ansiedad y angustia; y finalmente que la relación con tutores y tutoras es muy buena y continuaba incluso después de su incorporación a los grupos de referencia. Todas estas opiniones apoyan la idea de que el Aula de Enlace estaba funcionando como un espacio de acogida.

Respecto al último punto que se refiere a que los y las estudiantes continuaban la relación con las profesoras después de terminar el programa, una de las personas con las que trabajamos hizo mucho énfasis en este aspecto, sobre todo si permanecían en el mismo colegio, pero también cuando se escolarizaban en otro centro. Esta profesora utilizaba la expresión de que «no rompían el cordón umbilical» para referirse a estas relaciones. Esta metáfora nos remite a la significativa imagen de la clase como un útero que 
les «va a dar a luz» en el colegio una vez que se incorporen a las aulas de referencia, pero por otro lado implica una negación de su pasado escolar anterior en sus países de origen, como si estos chicos y chicas no tuvieran pasado, no tuvieran historia (Del Olmo 2007). Por otra parte, esta concepción de alumnos y alumnas sin pasado entra en contradicción con la utilización de sus lugares de origen y sistemas educativos previos para justificar las trayectorias posteriores (Cucalón Tirado 2015), tal y como analizaremos más adelante.

La idea del Aula de Enlace como espacio de acogida también está implícita en la manera en que el profesorado define su labor, dado que en su opinión no implica solamente enseñar castellano, sino un trabajo más "humano», que requiere empatía, "que te identifiques con ellos», "sentirte como se sienten ellos»:

Te piden una determinada actitud. Eso sí que lo sondearon bastante. Tener una actitud abierta, tolerante, que te preocupes por los chavales, porque la verdad es que cuando están en el Aula de Enlace eres como su padre y su madre. Siempre con ellos, cosa que yo no sé si hago pero consideraron que sí (Profesora de Aula de Enlace de un Colegio Concertado en Madrid, entrevista realizada por Pilar Cucalón Tirado, 9 jun. 2009).

porque no es un trabajo solo, aunque el principal trabajo sea aprender español, pero sí el afecto, la ayuda, el cariño para estar ahí, desde rellenar un impreso, que no tienen ni idea, paciencia toda. Debes tener mucha paciencia con ellos... (Profesora de Aula de Enlace de un Instituto de Enseñanza Secundaria en Parla, entrevista realizada por Pilar Cucalón Tirado, 11 jun. 2009).

El alumnado con el que trabajamos no solo reconoció la labor del profesorado en cuanto al aprendizaje del idioma, sino que señalaba la ayuda que recibía en cuestiones relacionadas con su vida escolar y personal. Sin embargo, las buenas relaciones que se establecen en el Aula de Enlace y el clima de acogida y cuidado encierra una paradoja, ya que entenderlo como espacio de acogida difumina, de alguna manera, los aspectos más académicos del programa, que son igualmente relevantes e incluso más de cara a las futuras trayectorias académicas. Esta se revela como una de las mayores preocupaciones tanto de docentes, como de estudiantes y sus familias, y constituye una de las tensiones a las que se enfrenta el profesorado del programa.

\section{LAS TRAYECTORIAS MÁS ALLÁ DEL AULA DE ENLACE}

Las trayectorias académicas de algunos alumnos y alumnas de las Aulas de Enlace son brillantes y con expectativas muy altas respecto a su futuro, por ello, consideran prioritarios los aspectos académicos y manifiestan su deseo de incorporarse al aula de referencia cuanto antes. A continuación, vamos a citar dos ejemplos recogidos en nuestros diarios de campo:

\begin{abstract}
Mariano es un chico de 16 años, de Rumanía, hace hoy justo tres meses que empezó el colegio, el mismo tiempo que lleva en España. Es muy inteligente, ha aprendido mucho español desde que le conozco, se aburre en clase y está deseando que le den el alta del Aula de Enlace. Toca el piano y el clarinete, vive en Villaverde y se queda a comer en el colegio un bocadillo, pero aprovecha para tocar el piano. Se incorporará a 40 de la ESO (Extracto del diario de campo de Margarita del Olmo, Colegio Concertado en Madrid, 23 ene. 2006).

Gao dice que ya han aprendido castellano y que ahora es buen momento para estar en una de las otras clases y aprender otras cosas, como matemáticas, inglés... Jing defiende lo mismo. El Aula de Enlace ha estado bien, les han gustado las profesoras porque no se enfadaban mucho con ellos pero dicen que cambiar está bien, aunque les da miedo suspender y no entender, porque quieren seguir aprendiendo cosas y sabiendo más (Extracto del diario de campo de Pilar Cucalón Tirado, Instituto de Enseñanza Secundaria en Parla, 28 may. 2009).
\end{abstract}

Como puede apreciarse en estos ejemplos hay estudiantes en el Aula de Enlace que manifiestan su deseo por salir del programa pero también expresan miedos por no poder comprender el idioma en las aulas de referencia. Esto muestra, a nuestro entender, que eran conscientes de que no habían adquirido todas las destrezas en la lengua necesarias para seguir las clases, aunque reconocieran la labor de sus docentes. Suárez-Orozco, Suarez-Orozco y Todorova (2008) llegan a la misma conclusión en su investigación comparando estudiantes migrantes en California y Massachusetts.

Pero no son solo los chicos y las chicas quienes reconocen la importancia de adquirir los conocimientos académicos, también el profesorado reclama la relevancia de esta dimensión, al considerar que el programa no aportaba a su alumnado las herramientas suficientes para desenvolverse en las aulas de referencia y argumentaba que, dada la organización del Aula de Enlace, su práctica educativa no podía 
llegar más lejos, puesto que lo que facilitaba era la enseñanza del castellano para desenvolverse en la vida cotidiana y la acogida.

Para alcanzar esta finalidad de incorporarse con éxito nos hemos encontrado con una docente que trataba de suplir los materiales que proporciona la Consejería para aprender castellano por otros que ella recopilaba, en sus propias palabras, "de acá y de allá»; es decir de cursos y asignaturas diferentes con los que elaboraba cuadernillos de "fichas» que iba adaptando al nivel de conocimientos y competencia en la lengua de manera individual. Ella concebía la enseñanza de la lengua en el contexto del aprendizaje académico.

Pero también con demandas concretas a la Consejería de Educación en relación a la implementación de medidas de apoyo posteriores a las Aulas de Enlace.

Nosotros pedimos soluciones: curso puente, horas de refuerzo post- aulas de enlace porque lo que nosotros vemos, donde cojea este programa es que mientras dure el programa, que tiene una atención bastante sistematizada, se les hace seguimiento, pero cuando pasa el Aula de Enlace, que es poco tiempo, ya no tienen apoyo. Algunas veces, la mayoría de las veces, sería necesario un apoyo. No tienen vocabulario curricular, ni base anterior. No hay mecanismo de apoyo a esto (Profesora 2 de Aula de Enlace de un Instituto de Enseñanza Secundaria en Parla, entrevista realizada por Pilar Cucalón Tirado, 11 jun. 2009).

Como puede apreciarse, esta profesora demanda apoyos posteriores al programa como una manera de responder a las necesidades de sus estudiantes, ya que los programas ya existentes de Diversificación o Compensatoria a los que suelen ser orientados a su salida del Aula de Enlace, no los considera adecuados porque no ofrecen una atención "sistematizada».

Tienen un conocimiento básico del idioma, pero después del Aula de Enlace ya no hay otro apoyo, más que los apoyos que hay para todos, los programas de compensatoria, diversificación, que no están adaptados a este tipo de alumnos [...] El alumno típico que provenga de los países orientales, de China, sus conocimientos matemáticos son muy altos, por encima de los conocimientos de los europeos. Sin embargo, desconocen todo sobre la historia de España, estos alumnos no son el perfil de diversificación para nada, pero si queremos que tengan una atención personalizada se les propone para ese programa. $Y$ en un grupo ordinario donde se sobreentiende que saben cosas anteriores en primaria, ellos se pierden y no hay nadie que les dé una formación entremedias, que les lance un puente entremedias para conocer toda esa información (Profesora 2 de Aula de Enlace en un Instituto de Enseñanza Secundaria en Parla, entrevista realizada por Pilar Cucalón Tirado, 11 jun. 2009).

El resto del profesorado de las escuelas que no pertenecía al Aula de Enlace se quejaba del nivel académico con el que el alumnado se incorporaba a los grupos de referencia y por ello abogaba por que se escolarizara en cursos inferiores a los que les correspondía por edad o las medidas especiales citadas (Compensatoria y Diversificación). Además, el claustro docente programaba visitas específicas para los chicos y chicas del Aula de Enlace a los programas de Garantía social ${ }^{21}$. Con esta afirmación lo que pretendemos es mostrar las diversas posturas ante las trayectorias escolares del alumnado migrante, pero todas ellas deben entenderse en relación con las presiones que los centros reciben en el marco de un sistema educativo que relaciona la presencia de estudiantado migrante con un nivel académico inferior. Aunque nosotras no hemos trabajado específicamente sobre este tema, nos parece importante señalar que la presencia de estudiantes migrantes influye en la elección de centro por parte de las familias y condiciona la práctica docente (Van Zanten 2007; Peláez Paz 2013).

A pesar de que el profesorado de Aula de Enlace considera inadecuados los programas de Diversificación y Compensatoria, acaba orientando a sus estudiantes a dichos programas, no solo ante la falta de alternativas, sino también porque, dadas sus características, ofrecen una atención más individualizada: tienen un número más reducido de estudiantes, se presiona menos en relación con los contenidos académicos y atribuyen al profesorado responsable una mayor empatía con su alumnado. Por este motivo, los perciben como un mal menor ante la falta de recursos adaptados al perfil del estudiantado de Aula de Enlace, que una vez que abandona el programa

21 Durante el trabajo de campo de Pilar Cucalón Tirado pasaron a llamarse Programas de Cualificación Profesional Inicial (PCPI). Tras la última ley de educación (LOMCE - Ley Orgánica para la Mejora de la Calidad Educativa-), los PCPI han sido extinguidos, poniéndose en marcha la llamada Formación Profesional Básica: <https://www.mecd. gob.es/educacion-mecd/mc/lomce/fp/fp-basica.html> (Fecha de acceso: 22 sept. 2017). 
es evaluado con los mismos parámetros que chicos y chicas que han nacido y vivido en la región.

En nuestra opinión el profesorado actúa de este modo con la finalidad de ofrecer a su alumnado mayores oportunidades y orientado por un ideal de buen y buena docente que moldea su práctica educativa. Downey (2015: 9) argumenta que profesores y profesoras se enfrentan simultáneamente a situaciones muy complicadas y a una narrativa cultural extendida que les exige sacar a cualquier joven adelante. Enfrentarse a esta paradoja es necesario para proteger una identidad de buen docente.

Creemos que esta idea es muy adecuada en nuestro contexto, ya que el profesorado con el que hemos trabajado también se ve afectado por esta paradoja: a pesar de la alta valoración que tienen de algunos y algunas de sus estudiantes, se encuentran en una situación estructural (de la que son en parte responsables) que impide a sus estudiantes continuar sus trayectorias en los itinerarios académicos que conducen a la universidad. Por este motivo concluimos que, para proteger su identidad de buenos docentes y la de la escuela como una institución facilitadora de igualdad de oportunidades, acaban resolviendo esta contradicción disminuyendo sus expectativas sobre la trayectoria de los y las jóvenes, y naturalizando las causas que hacen responsables de ello a factores externos al contexto educativo, tales como el origen cultural, el proceso migratorio, el sistema educativo de referencia y las condiciones familiares marcadas por la migración. A este respecto, Silvia Duschaztky y Carlos Skliar (2000), entre otros, señalan que esta forma de operar sobre la diversidad, en la que se culpa al diferente de los males que le acontecen, no solo tiene un papel fundamental a la hora anularlo psíquicamente, sino también a la hora de reforzar la propia identidad como exenta de esos males.

El conocimiento de la vida del alumnado que les proporciona el propio clima de cuidado que se desarrolla en el Aula de Enlace tiene, en nuestra opinión, este efecto negativo, ya que se utiliza para corroborar ideas preconcebidas sobre las dificultades de la migración o las familias de cara a los itinerarios. Es por este motivo por el que argumentamos que el buen clima se convierte en un arma de doble filo; dado que, por un lado, amortigua la llegada de los estudiantes pero, por el otro, ese conocimiento lleva a justificar la reconducción de las trayectorias académicas hacia los espacios periféricos de la escuela y del sistema educativo.
Este proceso tiene un efecto muy importante en el propio alumnado, ya que descienden sus expectativas y desechan los objetivos académicos con los que llegaron al comienzo del programa, que en muchos casos eran fruto de una escolarización muy exitosa en sus países de origen. Además nos parece fundamental tener en cuenta que, en algunos casos, el proceso migratorio ha sido planificado por la familia pensando en ofrecerles un mejor futuro a través de la educación. Aún así nos gustaría señalar que los chicos y chicas de las Aulas de Enlace, como los de cualquier clase, son muy diversas, al igual que sus familias, y hay quien va buscando únicamente lo que el programa le ofrece: suficiente competencia en castellano para insertarse en el mercado de trabajo.

Para ilustrar nuestra argumentación sobre el descenso de las expectativas por parte de algunos o algunas estudiantes, vamos a retomar la historia de Mariano ${ }^{22}$ porque creemos que muestra bien el proceso.

En la primera conversación que tuvo Margarita del Olmo cuando conoció a Mariano le dijo que estaba deseando dejar el Aula de Enlace para incorporarse al curso normal. Le contó que quería ir a la universidad a estudiar ingeniería, pero que aún no había decidido qué clase de ingeniería le interesaba más, aunque barajaba la posibilidad de hacer ingeniería informática. Durante los recreos, una de las cosas que hacían juntos era subir al último piso del colegio donde estaban colgadas las notas de Selectividad que pedía cada una de las facultades para entrar. Ingeniería informática era una de las carreras que exigía una nota más alta, un 8 , pero a Mariano no le preocupaba en absoluto. Se incorporó a 4. de la ESO poco tiempo antes de que terminara el curso escolar. Cada vez que se encontraba con él le veía rodeado de amigos, muchos ellos españoles, y notaba lo rápido que mejoraba su castellano. Tocaba el piano y los profesores del colegio habían conseguido que se matriculara en el conservatorio para estudiar clarinete. Al final del curso aprobó los exámenes.

El curso siguiente le encontró en el aula de 1.ำ de Bachillerato artístico y cuando le expresó su sorpresa por la opción elegida cuando su objetivo era hacer ingeniería informática. Le contó que había cambiado de idea sobre sus futuros estudios porque el colegio

22 Historia de vida construida a partir del diario de campo de Margarita del Olmo de los cursos 2005-2008. 
le había recomendado que no hiciera el Bachillerato científico, sino el artístico porque le iba a resultar más fácil ya que le gustaba la música. Le dijo que estaba contento y que pensaba hacer Bellas Artes. Durante todo el curso se vieron con frecuencia y hablaron de sus expectativas para el futuro. Casi al final le dijo que creía que no iba a conseguir entrar en Bellas Artes, así que subieron a ver el tablón de anuncios para ver qué nota pedían. Le dijo que no abandonara sus esperanzas porque podía conseguir fácilmente el 6 que requería. En ese momento no era posible distinguir en su acento ningún resto de su rumano.

La primera vez que le vio el tercer año había hecho ya la primera evaluación y había suspendido todas las asignaturas. Le dijo que estaba muy deprimido. Trató de animarle, pero no pudo. Acababa de aprobar el examen teórico del carnet de conducir (tenía ya 18 años), pero había suspendido el examen práctico. Le dijo que aunque su padre trataba de animarle y ayudarle, las clases prácticas de conducir le resultaban muy caras. Un tiempo después le dijo que había aprobado el examen para pertenecer a una orquesta municipal, pero que ello implicaba un montón de trabajo extra y estaba pensando en dejar la escuela. Le dijo que estaba buscando un trabajo como DJ en una discoteca. Cuando le recordó sus expectativas de ir a la Universidad, dijo que estaba pensando en olvidarse del plan y hacer Formación Profesional. Había pensado en estudiar para convertirse en ingeniero de sonido. La última vez que se encontró con él estaba a punto de conseguir empleo en una empresa discográfica para hacer pequeños trabajos, pero luego supo que había abandonado el colegio, y más tarde que la empresa que le iba a contratar, había cerrado por falta de trabajo. Nunca más volvió a verle.

Historias como las de Mariano no son excepcionales. En el trabajo de campo de Pilar Cucalón Tirado se dieron casos semejantes, fue muy significativo el de una estudiante de China, que con notas excelentes en su país de origen y deseos por estudiar medicina, acabó abandonando su escolarización sin terminar la secundaria obligatoria.

A lo largo de nuestros trabajos de campo, las docentes de las Aulas de Enlace nos han manifestado preocupación cuando sus estudiantes sufrían porque sus calificaciones académicas disminuían respecto a sus trayectorias académicas previas:

Hay alumnos que vienen con un expediente académico brillante y no van a aceptar el por debajo del brillante. Entonces, cuando vienen con un notable vienen llorando al Aula de Enlace. Estoy poniendo casos extremos pero casos que hemos vivido, nombres puedo dar de este año que venían con esas notas y claro que no lo aceptan. Y de ahí, para abajo, todo (Profesora 2 de Aula de Enlace de un Instituto de Enseñanza Secundaria en Parla, entrevista realizada por Pilar Cucalón Tirado, 11 jun. 2009).

La misma profesora reconoce que se hace repetir curso varias veces y/o que se orienta al estudiantado hacia los grupos de diversificación. Estas situaciones, según dicha docente, son difíciles de asumir por parte de quienes desean ir a la Universidad.

\begin{abstract}
Hay alumnos que lo viven mejor, porque han sabido hacer que estas cosas no les afecten tanto, pero realmente tienen que afectar. Si un alumno quiere realmente sacarse una titulación les tiene que afectar. Les tiene que afectar que vienen aquí, que por la edad se les matricule en tercero, vuelvan a hacer tercero al año siguiente y ya entren en tercero de diversificación. Claro, ya son muchos años y ellos quieren seguir estudiando y les cuesta (Profesora 2 del Aula de Enlace en un Instituto de Enseñanza Secundaria en Parla, entrevista realizada por Pilar Cucalón Tirado, 11 jun. 2009).
\end{abstract}

La reflexividad de esta docente sobre su práctica, que también la hemos apreciado en otros casos, les lleva a plantear la necesidad de que existan ayudas o programas posteriores al Aula de Enlace, como hemos mencionado anteriormente, pero también a tratar de solucionar la situación trabajando con los chicos y chicas y sus familias ${ }^{23}$ (Jociles, Franzé y Poveda 2012a).

ya te he dicho, he visto llorar a alumnas que sacaban menos notas de lo que estaban acostumbradas en su país de origen. Bueno, nosotros formamos a los padres primero para quitar la presión, si es por ahí la presión, hay veces que sí y otras que no. Los padres son los que quieren que salgan del Aula de Enlace y ¿estas notas qué? Los padres suelen ser bastantes receptivos. Les decimos póngase usted

23 En este momento nos parece significativo apuntar que el hecho de que nosotras pudiéramos investigar en esas Aulas de Enlace no solo era consecuencia de que sus docentes estuvieran interesadas en ayudarnos para que nuestros trabajos salieran adelante, sino que pretendían que tuviera visibilidad y legitimidad en el campo académico la labor que llevaban a cabo en una medida tan controvertida. Además, estaban interesadas en que presenciáramos algunas de las dificultades a las que se enfrentaban y que transmitiéramos sus reclamaciones, especialmente en relación a los aspectos en los que consideraban que el programa flojeaba, como era el paso a las aulas de referencia. 
en el aula y vea la presión que tienen (Profesora 2 del Aula de Enlace en un Instituto de Enseñanza Secundaria en Parla, Entrevista realizada por Pilar Cucalón Tirado, 11 jun. 2009).

En nuestra opinión, este intento de que algunas familias relajen la «presión» contrasta con la imagen de descuido, desatención y falta de responsabilidad que en otras ocasiones les atribuyen en relación con la trayectoria escolar de sus hijos e hijas.

\begin{abstract}
Me explica que en Parla hay un fracaso escolar muy fuerte, que él no sabe qué se puede hacer para solucionarlo, pero que existe como tal, que hay padres que es muy complicado dar con ellos porque trabajan todo el día y que en el caso de los inmigrantes pues es "que estos señores vienen para otra cosa» (Extracto del diario de campo de Pilar Cucalón Tirado, Instituto de Enseñanza Secundaria en Parla, 7 may. 2009).
\end{abstract}

Una falta de atención que en el caso de las familias extranjeras atribuyen a diferencias "culturales» muy acentuadas y/o las dificultades de carácter económico, cuyas formas de organización son vividas por el profesorado como "raras» o, en nuestra interpretación, distantes de un modelo familiar en el que ambos padres con recursos económicos suficientes conviven con sus hijos e hijas en una casa:

Familias muchas veces inexistentes, de chinos que no sabes si viven con su tío, con su tía porque hay un maremágnum de la sociedad china que no nos enteramos de lo que pasa, o mienten, esconden por miedo. Indudablemente, familias muy raras. Situaciones económicas tremendas. El padre en Marruecos y la madre en España trabajando [...] A veces la realidad es tan difusa, pues son padres que, o solo está el padre o la madre no, o están viviendo con los tíos porque los padres vendrán más adelante. Ha habido alumnas, ha habido alguna chica que estaba sola en Madrid, que ni si quiera tenía familiares y que se tenía ella que organizar de manera única. Hay algún otro alumno que estaba en un albergue. Entonces, hay familias que tienen un drama humano detrás, supongo, que tienen pocos recursos, muy pocos recursos y entonces ha habido dificultades para entendernos, para entenderles, porque no conocían el idioma como te digo y tampoco estaban en un sitio privilegiado, sino conviviendo con muchos inmigrantes porque no tenían un lugar. A veces, las diferencias culturales son tan marcadas (Profesora del Aula de Enlace de un Instituto de Enseñanza Secundaria en Parla, entrevista realizada por Pilar Cucalón Tirado, 2 jul. 2009).
En el caso de los y las alumnas migrantes, tanto dentro como fuera de las Aulas de Enlace, las expectativas del profesorado hacia sus estudiantes no solo están influidas por las características socioeconómicas y migratorias de las familias, sino que los sistemas educativos de los lugares de los que proceden también se utilizan como ideas para justificar trayectorias educativas fuera de los itinerarios que llevan a la Universidad (Franzé, Moscoso y Calvo 2010; Jociles, Franzé y Poveda 2012b). En este sentido, nos gustaría señalar que en los discursos son agrupados en grandes bloques geopolíticos y apreciados de manera diferente, de manera que son mucho mejor valorados los de "Europa del Este» y "China» frente a los de "Latinoamérica» y "África», sin mostrar diferencias entre estos grandes bloques. En nuestros trabajos de campo, sin embargo, hemos apreciado mucha mayor diversidad entre las experiencias de los y las estudiantes. A la luz de estas ideas, nos parece importante señalar que la escuela está produciendo una valoración más positiva de determinados orígenes, muy semejante al concepto de "minoría modelo» desarrollado en Estados Unidos en relación con personas de origen asiático-americano (Suzuki 1977 y 1989). En el estado español, esta idea ha sido trabajada especialmente en Cataluña (Bereményi 2011).

Además de los argumentos citados anteriormente, queremos añadir que la "cultura» (Franzé, Moscoso y Calvo 2010; Jociles, Franzé y Poveda 2012b) entendida por los distintos actores como estilo de sociabilidad e identificada con los países o a las regiones geopolíticas de las que acabamos de hablar, también es considerada por los y las docentes como un factor determinante para explicar las trayectorias de nuestros estudiantes, dado que, en su opinión, estos estilos de sociabilidad condicionan el aprendizaje del castellano, tanto a la hora de comunicarse en las relaciones cotidianas como en términos académicos.

Para concluir deseamos señalar que entrelazar nuestros trabajos de campo nos ha permitido analizar con mucha mayor complejidad los argumentos que el profesorado utiliza para justificar esta desigualdad, pero también nos parecía necesario comprender las múltiples tensiones a las que se enfrenta su práctica. Para ello nos ha parecido importante prestar atención en ese artículo a los vaivenes en la normativa que regula el programa, el camino hacia la privatización de la enseñanza, y la presión de los ideales de «buen docente» y de la escuela como igualadora de oportunidades que moldea su práctica. Todo esto nos ha 
posibilitado presentar aquí un análisis longitudinal del día a día de la práctica educativa en un recurso como las Aulas de Enlace y de las lógicas que la sustentan, constituyendo como intrínseca a la (bienintencionada) acogida del alumnado migrante la producción de trayectorias académicas desiguales. De tal modo que los casos de antiguos estudiantes de las Aulas de Enlace que llegan a la Universidad a pesar de sus expectativas iniciales e historias escolares previas acaban siendo excepcionales.

Desde nuestro punto de vista, se hace especialmente urgente algo que ya han visibilizado otros análisis y otras perspectivas: dejar de entender la diversidad de los estudiantes como un déficit, que es la lógica que rige este tipo de programas educativos y todas las medidas inspiradas en la idea de compensación; pero también, como expresó de manera explícita uno de los docentes con los que trabajamos, es necesario reclamar el derecho de los y las estudiantes migrantes a la educación.

\section{BIBLIOGRAFÍA CITADA}

Aguado, Teresa, Patricia Mata e Inés Gil-Jaurena. 2017. «Mobilizing Intercultural Education for Equity and Social Justice. Time to React against the Intolerable: A Proposal from Spain». Intercultural Education 28: 408-423. doi: <https:// doi.org/10.1080/14675986.2017.1333874>.

Alegre, Miquel Ángel, Ricard Benito y Sheila González. 2008. De l'aula d'acollida a l'aulaordinària: Processos d'escolarització de l'alumnat estranger. Bellatera: Institut de Govern i Polítiques Públiques.

Antolínez, Inmaculada. 2015. «Evolución de las políticas educativas en Andalucía para la atención a alumnado extranjero desde una mirada intercultural crítica: el caso de las aulas temporales de adaptación lingüística», en Francisco Javier García Castaño, Adelaida Megías y Jennifer Ortega (eds.), Actas del VIII Congreso sobre Migraciones Internacionales en España (Granada, 16-18 de septiembre de 2015): S02/79-S02/91. Granada: Instituto de Migraciones.

Ávila, Débora y Marta Malo. 2007. «¿Quién puede habitar la ciudad? Fronteras, gobierno y transnacionalidad», en Observatorio Metropolitano (ed.), Madrid: ¿la suma de todos? Globalización, territorio y desigualdad: 505-534. Madrid: Traficantes de Sueños,

Bereményi, Bálint-Abel. 2011. «Cuando la etnicidad supedita la nacionalidad. Complicando la noción de minoría modelo entre inmigrantes "del Este" en España», en Franciso Javier García Castaño y Nina Kressova (coords.), Actas del I Congreso Internacional sobre Migraciones en Andalucía: 16191627. Granada: Instituto de Migraciones.

Boyano, Manuela, José Luis Estefanía, Henedina García y Montserrat Homedes. 2006. Aulas de Enlace: orientaciones metodológicas y para la evaluación. Madrid: Comunidad de Madrid.

Carbonell, Fracesc. 2006. L'acollida. Acompanyamen d'alumnat nonvingut. Barcelona: Eumo editorial/Jaume Bofill.

Carrasco, Silvia. 2011. «Segregación escolar e inmigración: repensando planteamientos y alternativas», en Francisco Javier García Castaño y Silvia Carrasco Pons (eds.), Población inmigrante y escuela: conocimientos y saberes de investigación: 271-287. Madrid: Ministerio de Educación.

Carrasco, Silvia y Margaret Gibson. 2010. «La educación de los jóvenes de origen inmigrante en secundaria: algunas lecciones comparativas desde Cataluña y California». Revista del Ministerio de Trabajo e Inmigración 90: 9-76.

Carrasco, Silvia, Jordi Pàmies y Laia Narciso. 2011 «Políticas de inclusión educativa e inmigración: la paradoja de los "Espacios de Bienvenida Educativa"», en Luis Díaz Viana, Óscar Fernández Álvarez y Pedro Tomé (coords.), Lugares, tiempos, memorias: la antropología ibérica en el siglo XXI: 26152624. León: Universidad de Léon.

Castilla, José. 2015. «Las ATAL: trayectoria histórica y gramáticas de identidad/alteridad», en Francisco Javier García Castaño, Adelaida Megías y Jennifer Ortega (eds.), Actas del VIII Congreso sobre Migraciones Internacionales en España (Granada, 16-18 de septiembre de 2015): S02/92-S02/102. Granada: Instituto de Migraciones.

Consejería de Educación. 2008. Instrucciones de la Viceconsejería de educación de la Comunidad de Madrid por las que se regulan la escolarización y las aulas de enlace para el alumnado proceden de sistemas educativos extranjeros. Madrid: Consejería de Educación, Comunidad de Madrid.

Consejería de Educación. 2009. Datos y cifras de la Educación 08/09. Madrid: Comunidad de Madrid.

Consejería de Educación. 2010a. Datos y cifras de la Educación 2009/2010. Madrid: Comunidad de Madrid.

Consejería de Educación. 2010b. Datos y cifras de la Educación 2010/2011. Madrid: Comunidad de Madrid.

Consejería de Educación y Empleo. 2012. Datos y cifras de la Educación 2011/2012. Madrid: Comunidad de Madrid.

Consejería de Educación, Juventud y Deporte. 2013. Datos y cifras de la Educación 2012/2013. Madrid: Comunidad de Madrid.

Consejería de Educación, Juventud y Deporte. 2014. Datos y cifras de la Educación 2013/2014. Madrid: Comunidad de Madrid.

Consejería de Educación, Juventud y Deporte. 2015. Datos y cifras de la Educación 2014/2015. Madrid: Comunidad de Madrid.

Consejería de Educación e Investigación. 2017. Datos y cifras de la Educación 2017/2018. Madrid: Comunidad de Madrid.

Cucalón Tirado, Pilar. 2015. Tránsitos, límites y migrantes en las escuelas: una investigación en las aulas de enlace de la Comunidad de Madrid. Madrid: Universidad Complutense. 
Cucalón Tirado, Pilar y Margarita del Olmo. 2010. «Redefiniendo trayectorias escolares. Las aulas de enlace en la Comunidad de Madrid». Revista de la Asociación de Sociología de la Educación 3(2): 223-233.

Del Olmo, Margarita. 2007. «La articulación de la diversidad en la escuela: Un proyecto de investigación en curso sobre las "Aulas de Enlace"». Revista de Dialectología y Tradiciones Populares 62(1): 187-203.

Del Olmo, Margarita. 2009. «Un análisis critico de las “Aulas de Enlace" como medida de integración», en Matilde Fernández Montes y Walltraud Müllauer-Seichter (eds.), La integración escolar a debate: 170-181. Madrid: Pearson.

Del Olmo, Margarita. 2010. Re-Shaping Kids through Public Policy on Diversity. Lessons from Madrid. Viena: Navreme.

Dirección General de Mejora de la Calidad de la Enseñanza (ed.). 2011. Datos y cifras de la educación 2011-2012. Madrid: Comunidad de Madrid, Dirección General de Mejora de la Calidad de la Enseñanza. Disponible en: <http://www. madrid.org/bvirtual/BVCM016051.pdf>. Fecha de acceso: 11 oct. 2018.

Downey, C. Aiden. 2015. “"They Kicked Him Out”: Teachers' Students Stories as Counterstories». Anthropology and Education Quarterly 46(1): 1-18.

Duschatzky, Silvia y Carlos Skliar. 2000. "La diversidad bajo sospecha. Reflexiones sobre los discursos de la diversidad y sus implicaciones educativas», en Por la inclusión. Un espacio para pensar la inclusión y la igualdad educativa desde el Mercosur. Disponible: en <www. porlainclusionmercosur.educ.ar/documentos/Ladiversidadbajosospecha.pdf>. Fecha de acceso: 22 sept. 2017.

Escarbajal Frutos, Andrés. 2010. «Estudio de las respuestas educativas ofrecidas a los inmigrantes en la región de Murcia». Revista de investigación educativa 28(3): 149-164.

Etxeberria, Felix, Hilario Murua, Joxe Garmendia y Juan Etxeberria. 2015. «Evaluación de los dispositivos de acogida para el alumnado inmigrante en Euskadi», en Francisco Javier García Castaño, Adelaida Megías y Jennifer Ortega (eds.), Actas del VIII Congreso sobre Migraciones Internacionales en España (Granada, 16-18 de septiembre de 2015): S02/54S02/66. Granada: Instituto de Migraciones.

Franzé, Adela, María Fernanda Moscoso y Albano Calvo. 2010. «Donde nunca hemos llegado. Alumnado de origen latinoamericano: entre la escuela y el mundo laboral», en Grupo Interdisciplinario Investigador@s migrantes (eds.), Biculturalismo y segundas generaciones. Integración social, escuela y bilingüismo: 123-138. Madrid: Instituto de Estudios Políticos para América Latina y África, IEPALA.

García Castaño, Francisco Javier, José Castilla y Lorenzo Capellán de Toro. 2015. "¿Compensar es igualar?: trayectorias de escolares de nacionalidad extranjera en dispositivos "especiales" en el sistema educativo andaluz», en Francisco Javier García Castaño, Adelaida Megías y José Ortega Torres (eds.), Actas del VIII Congreso sobre Migraciones Internacionales en España (Granada, 16-18 de septiembre de 2015): S02/13-S02/28. Granada: Instituto de Migraciones.
García Castaño, Francisco Javier, María Rubio Gómez y Ouafaa Bouachra. 2011. "Población inmigrante y escuela en España: un balance de investigación», en Francisco Javier García Castaño y Silvia Carrasco Pons (eds.), Población migrante y escuela: conocimientos y saberes de investigación: 143-233. Madrid: Ministerio de Educación.

Jiménez, Rafael, Mayka Cotrina, Concepción García Romero. 2011. "Las Aulas Temporales de Adaptación Lingüística (ATAL) en la provincia de Cádiz. Un informe de investigación», en Francisco Javier García Castaño y Silvia Carrasco (eds.), Población inmigrante y escuela: conocimientos y saberes de investigación: 615-659. Colección Estudios Creade 8. Madrid: Ministerio de Educación de España.

Jociles, Maribel, Adela Franzé y David Poveda. 2012a. «La construcción de la desigualdad educativa en educación secundaria: el papel del departamento de orientación», en Francisco Javier García Castaño y Antonia Olmos (eds.), Segregaciones y construcción de la diferencia en la escuela: 183-199. Madrid: Trotta.

Jociles, Maribel, Adela Franzé y David Poveda. 2012b. «La diversidad cultural como problema: representaciones y prácticas escolares con adolescentes de origen latinoamericano». Alteridades 22(43): 63-78.

Laval, Christian. 2005. «Per què l'escola no és una empresa?». Debats d'educació. Barcelona: Fundació Jaume Bofill/UOC. Disponible en: <http://www.uoc.edu/dt/cat/laval0505.pdf>. Fecha de acceso: 1 feb. 2012.

Mijares Molina, Laura. 2003. «Viejos esquemas para nuevas situaciones: gestión y contradicciones del programa de enseñanza de lengua y cultura de origen (ELCO) marroquí». Suplementos OFRIM 10: 47-63.

Ortiz Cobo, Mónica. 2011. «Enseñanza de la lengua en contextos españoles de acogida. El caso de las Aulas Temporales de Adaptación Lingüística (ATAL)», en Francisco Javier García Castaño y Silvia Carrasco Pons (eds.), Población migrante y escuela: conocimientos y saberes de investigación: 661694. Madrid: Ministerio de Educación.

Peláez Paz, Carlos. 2013. «Elección de centro escolar: la construcción de la desigualdad y estratificación social en Madrid». Ponencia presentada al XI Congreso español de Sociología.

Pérez Milans, Miguel. 2007. «Las aulas de enlace: un islote de bienvenida», en Luisa Martín Rojo y Laura Mijares, Voces del Aula. Etnografías de la Escuela Multilingüe: 111-146. Colección Estudios CREADE n. 3. Madrid: Centro de Investigación y Documentación Educativa, CIDE.

Poveda, David, Maribel Jociles y Adela Franzé. 2009. «La diversidad cultural en la Educación Secundaria en Madrid: Experiencias y Prácticas institucionales con alumnado inmigrante latinoamericano». Revista Papeles de Trabajo sobre Cultura, Educación y Desarrollo Humano 5(3): [s. p.].

Poveda, David, Adela Franzé, María Isabel Jociles et al. 2011. "La segregación étnica en la educación secundaria de la ciudad de Madrid: un mapa y una lectura crítica», en Francisco Javier García Castaño y Silvia Carrasco Pons (eds.), Población migrante y escuela: conocimientos y saberes de investigación: 291-316. Madrid: Ministerio de Educación. 
Suárez-Orozco, Carola, Marcelo Suárez-Orozco y Irina Todorova. 2008. Learning a New Land. Immigrant Students in American Society. Cambridge: Harvard University Press.

Suzuki, Bob H. 1977. "Education and the Socialization of Asian Americans: A Revisionist Analysis of the "Model Minority" Thesis». Amerasia Journal 4(2): 23-51.

Suzuki, Bob H. 1989. "Asian Americans as the "Model Minority": Outdoing Whites? Or Media Hype?». Change. The Magazine of Higher Learning 21(6): 13-19. doi: <https://doi.or $\mathrm{g} / 10.1080 / 00091383.1989 .9937601>$.

Tobarra, Sebastián. 2006. "Compensar las desigualdades». El País 11 sep. Disponible en: <https://elpais.com/dia- rio/2006/09/11/educacion/1157925603 850215.html>. Fecha de acceso: 15 oct. 2018

Van Zanten, Agnès. 2007. "Reflexividad y elección de la escuela por los padres de la clase media en Francia». Revista de Antropología Social 16: 245-278.

Vila, Ignasi, Imma Canal, Pere Mayan, Santiago Perera, Josep María Serra, Carina Siqués. 2009 «Las aulas de acogida de la Educación Primaria de Cataluña el curso 2005-2006: sus efectos sobre el conocimiento del catalán y la adaptación escolar». Revista Infancia y Aprendizaje: Journal for the Study of Education and Development 32(3): 307-327. 